\title{
Quantitative Trait Loci Controlling the Number of Spikelets and Component Traits in Rice: Their Main Effects and Interaction with Years
}

\author{
Tsuneo Kato*1) \\ School of Bioresources, Hiroshima Prefectural University, 562 Shobara, Hiroshima 727-0023, Japan \\ 1) Present address: School of Biology-Oriented Science and Technology, Kinki University, 930 Nishimitani, Uchita, Wakayama 649- \\ 6493, Japan
}

To develop superior rice panicles with a sufficient sink capacity and a high degree of grain filling, we should consider the within-panicle distribution of spikelets showing different degrees of grain filling, as well as the total spikelet number. In the present study, several component traits of the number of spikelets per panicle which are related to the within-panicle distribution, and also the number of panicles per plant, were examined using 68 recombinant inbred lines (RILs) derived from a cross of Asominori/IR24 grown over a period of four years, 1997, 1998, 2000 and 2001. For every trait related to the spikelet number per plant, analyses of variance (ANOVA) were conducted at the 375 position of molecular markers one by one to detect significant main effects of quantitative trait loci (QTLs) and interactions of marker genotype and year of cultivation (QY interactions). Then, genetic associations of these traits were analyzed by examining the positions of the relevant genome regions which showed significant main effects and/or interactions for different traits. Finally, the QTLs causing favorable genetic effects and associations for the development of superior panicles were surveyed. The results showed that most of the genome region identified to increase the spikelet number per panicle also increased the number of spikelets on the secondary branches which are characterized by a low degree of grain filling. One region on chromosome 3, on the other hand, increased the spikelet number per panicle through the increase of the number of spikelets on primary branches which generally show a high degree of grain filling. Significant QY interactions were demonstrated in many genome regions for all traits, whereas the interactions of RIL and year could not be detected in an overall ANOVA in which the marker genotypes of RILs were not being considered. The region on chromosome 3, cited above, did not exhibit a significant QY interaction. This region appears to involve QTL(s) suitable for the development of superior panicle types.

Communicated by H. Ikehashi

Received September 10, 2003. Accepted January 27, 2004.

*Corresponding author (e-mail: tkato@bio.waka.kindai.ac.jp)
Key Words: rice, spikelet number, component traits, genetic association, main effect, quantitative trait locus (QTL), QTL-by-year interaction.

\section{Introduction}

To contribute to the development of high-yielding rice cultivars, we previously examined the genetic association of traits related to the yield sink capacity and the degree of grain filling (Kato 1996, Kato and Takeda 1996, Kato 1997). These studies suggested that it should be possible to develop a novel genotype with numerous spikelets in a panicle (extra-heavy panicle genotype) and which also shows a high degree of grain filling. The proposed genotype could be developed by increasing the number of primary branches (PBs) which generally bear spikelets that are superior in terms of grain filling. Simultaneously, in this genotype overproduction of secondary branches (SBs) which bear inferior spikelets should be avoided (Kato and Takeda 1996). The proposed concept is of great significance for the development of high-yielding rice cultivars (Khush 1996, Peng et al. 1999), because existing cultivars of the extra-heavy panicle type often show a low degree of grain filling (Yamamoto et al. 1991, Peng et al. 1999, Yang et al. 2002).

In previous studies (Kato and Takeda 1996, Kato 1997), genetic correlation and correlated selection responses were examined for the traits related to the yield sink capacity to evaluate their genetic relationships. In the present study, the analysis of quantitative trait loci (QTLs) was conducted for multiple traits that control the spikelet number per plant which is a primary character of the yield sink capacity and grain yield (Feil 1992). In addition, the genome regions which showed significant effects on different traits, particularly between a compound trait and component traits, and caused genetic associations of these traits.

In the present study, the interaction of marker genotype and years of cultivation [hereafter referred to QTL-by-year (QY) interaction] at every marker position for traits related to the spikelet number per plant were analyzed. The genome regions showing a significant $\mathrm{QY}$ interaction for a compound trait were tested for significant QY interactions regarding the components of the compound trait. The QTL 
main effects and QY interactions for multiple traits and their relationship, as well as for a single trait, could provide valuable information for the development of high-yielding rice cultivars with improved panicle-sink architecture.

\section{Materials and Methods}

\section{Plant material}

Sixty-eight recombinant inbred lines (RILs) derived from the $\mathrm{F}_{2}$ individuals of an indica-japonica cross between two rice (Oryza sativa L.) cultivars, Asominori (japonica type) and IR24 (indica type), were used in the present study. They were kindly supplied by Prof. A. Yoshimura, Kyushu University, Japan.

\section{Field trials}

In 1997, 1998, 2000 and 2001, the RILs were grown at Hiroshima Prefectural University, Shobara, Hiroshima, Japan. The average air temperatures and total number of sunshine hours during August in each year, in which most of the RILs flowered, were $24.5^{\circ} \mathrm{C}$ and $177.6 \mathrm{~h}(1997), 24.9^{\circ} \mathrm{C}$ and $131.7 \mathrm{~h}(1998), 24.9^{\circ} \mathrm{C}$ and $178.0 \mathrm{~h}(2000)$, and $24.4^{\circ} \mathrm{C}$ and $178.0 \mathrm{~h}$ (2001), respectively. Seeds were sown in nursery trays each year at the end of April, and were transplanted in a paddy field about 40 days later at a density of one plant per hill. The distance between plants in a row was $15 \mathrm{~cm}$, and that between rows of plants was $30 \mathrm{~cm}$ apart. Each RIL was cultivated in a plot containing 24 plants that were arranged in the paddy field according to a randomized block design with two replications. The total amount of fertilizer applied was $10 \mathrm{~g} \mathrm{~m}^{-2}$ each for nitrogen, phosphorus and potassium. RILs were cultivated according to common practices routinely applied in the northern part of Hiroshima prefecture, including pest management.

After ripening time, the number of panicles per plant (B in Table 1, etc.) was counted in all the plants and one panicle from the tallest culm of each plant was harvested. In each panicle, the following characters were determined; the number of spikelets (excluding the spikelets on SBs) on PBs (C1), the number of spikelets on SBs (C2), the number of PBs per panicle $\left(\mathrm{Cl}_{1}\right)$ and the number of SBs per panicle $\left(C 2_{1}\right)$. The numbers of shattered, imperfectly filled, and unfertilized spikelets were included in the number of spikelets counted. The number of spikelets per $\mathrm{PB}\left(\mathrm{Cl}_{2}=\mathrm{C} 1 / \mathrm{C}_{1}\right)$, the number of spikelets per $\mathrm{SB}\left(\mathrm{C} 2_{2}=\mathrm{C} 2 / \mathrm{C} 2_{1}\right)$, the total number of spikelets per panicle $(\mathrm{C}=\mathrm{C} 1+\mathrm{C} 2)$, and the number of spikelets per plant $(\mathrm{A}=\mathrm{B} \times \mathrm{C})$ were calculated.

\section{Data analysis}

Two kinds of analysis of variance (ANOVA) were conducted for every trait observed. The first one was an overall ANOVA (two-way classification) with two replications, in which the information about the markers was not taken into account. Two sources of variance, RIL and year, were considered to be random effects. The second one was conducted after the 68 RILs were classified into two marker-genotype groups, Asominori-type and IR24-type, for each of the 375 molecular markers. These markers had already been mapped onto 12 chromosomes in the same set of RILs used in this study (Tsunematsu et al. 1996; the marker genotypes of the RILs are exhibited at http://rgp.dna.affrc.go.jp/dnabank/ publicdata/asoir24/asoIR24r.html). The number of RILs tested varied depending on the markers, because some marker data were not available for a few RILs. The sum of squares (SS) for RILs, which was calculated from the first ANOVA, was divided into two parts according to an orthogonal comparison (Snedecor and Cochran 1967). One part included the SS for the comparison between the two groups [one degree of freedom (df)]. This source of variance was considered to be a fixed effect. The other part corresponded to the residual SS within the RIL's SS, which was obtained by subtracting the comparison's SS from the RIL's SS. In the same way, the SS for the interaction of RIL and year of cultivation was divided into an orthogonal comparison part (3 df) and a residual part within the interaction's SS. The QTL main effect and QY interaction were assessed by an Ftest at the 0.01 probability level. In this F-test for the main effect and interaction, the comparison's mean squares were divided by the residual mean squares within the respective factors. When significant main effects or interactions were detected in a single marker or several successive markers, the term genome "region" was used at these marker positions where at least one common QTL was assumed to exist. Also, when different traits were shared by at least one marker in their regions, a common name (number) was given for these regions.

To evaluate the genetic association between the traits described above, genetic correlation coefficients, i.e. the ratios of the genetic component of covariance between two traits to the geometric mean of the genetic components of variance of the two traits, were calculated (Kato and Takeda 1996).

\section{Results}

\section{Overall analysis of variance}

The overall ANOVA, in which RIL and year of cultivation were sources of variation, revealed that all the traits considered showed a significant variation between years and between RILs (Table 1). On the other hand, no traits except for one (number of PBs per panicle) showed a significant interaction of RIL and year (Table 1).

\section{Main effects of QTLs}

Table 2 depicts the second ANOVA, in which the orthogonal comparison between marker genotypes and its residual were additional variance sources. Figure 1 illustrates the detected genome regions along 12 chromosomes which showed significant main effects for the traits examined, and therefore appeared to contain one or more QTLs. Table 3 summarizes the significant F-values for these genome regions. These figure and table also show a coincidence profile 
Table 1. ANOVA for traits related to rice spikelet number per plant according to a two-way classification in which RILs and years of cultivation are considered to be random effects

\begin{tabular}{|c|c|c|c|c|c|}
\hline \multirow{2}{*}{ Trait } & \multirow{2}{*}{ Mean } & \multicolumn{4}{|c|}{ Mean square } \\
\hline & & Year $(3)^{2)}$ & RIL (67) & Interaction (201) & Residual (271) \\
\hline Number of spikelets plant ${ }^{-1}\left(\times 10^{-5}\right)^{1)}(\mathrm{A})$ & 1855.2 & $181.12 * *$ & $13.46^{* *}$ & 1.21 & 1.20 \\
\hline Number of panicles plant ${ }^{-1}(\mathrm{~B})$ & 13.9 & $672.05 * *$ & $52.81 * *$ & 3.30 & 3.57 \\
\hline Number of spikelets panicle ${ }^{-1}(\mathrm{C})$ & 134.5 & $4903.33^{* *}$ & $5948.11 * *$ & 296.39 & 371.38 \\
\hline Number of spikelets on PBs (C1) & 66.8 & $2724.92 * *$ & $743.71 * *$ & 25.67 & 21.68 \\
\hline Number of PBs panicle ${ }^{-1}\left(\mathrm{Cl}_{1}\right)$ & 11.5 & $43.98 * *$ & $19.57 * *$ & $0.56^{* *}$ & 0.46 \\
\hline Number of spikelets $\mathrm{PB}^{-1}\left(\mathrm{Cl}_{2}\right)$ & 5.8 & $1.66^{* *}$ & $0.74 * *$ & 0.05 & 0.03 \\
\hline Number of spikelets on SBs (C2) & 67.7 & $1060.83 * *$ & $5286.58 * *$ & 227.86 & 293.28 \\
\hline Number of SBs panicle ${ }^{-1}\left(\mathrm{C} 2_{1}\right)$ & 22.2 & $100.83 * *$ & $442.33 * *$ & 17.40 & 23.25 \\
\hline Number of spikelets $\mathrm{SB}^{-1}\left(\mathrm{C}_{2}\right)$ & 3.0 & $0.52 * *$ & $0.32 * *$ & 0.03 & 0.02 \\
\hline
\end{tabular}

1) Mean squares listed are actual values $\times 10^{-5}$.

2) Values in the parenthesis indicate the degrees of freedom for the respective sources.

**: indicates that the mean square is statistically significant at the 0.01 probability level.

Table 2. Examples of ANOVA for the number of rice spikelets on SBs at the positions of some markers successively located on chromosome 1 , with one region defined between R3202 and C86 for this trait

\begin{tabular}{|c|c|c|c|c|c|c|c|}
\hline \multirow{2}{*}{ Source } & \multirow{2}{*}{$\mathrm{df}^{1)}$} & \multicolumn{6}{|c|}{ Mean square ${ }^{2)}$ at } \\
\hline & & $\mathrm{XNpb54}$ & R3203 & G2200 & $\mathrm{XNpb113}$ & $\mathrm{C} 86$ & XNpb93 \\
\hline Year $(Y)$ & 3 & $10.61 * *$ & $9.87 * *$ & $9.87 * *$ & $8.07 * *$ & $9.74 * *$ & $7.91 * *$ \\
\hline RIL (R) & $61-67$ & $52.87 * *$ & $52.20 * *$ & $52.20 * *$ & $50.48 * *$ & $52.94 * *$ & $51.21 * *$ \\
\hline Comparison $^{3)}$ & 1 & 281.04 & $377.18 * *$ & $538.09 * *$ & $421.71 * *$ & $372.82 * *$ & 247.83 \\
\hline Residual $^{4)}$ & $60-66$ & 49.41 & 47.20 & 44.72 & 44.40 & 47.94 & 47.93 \\
\hline Interaction $(\mathrm{Y} \times \mathrm{R})$ & $183-201$ & 2.28 & 2.28 & 2.28 & 2.13 & 2.30 & 2.14 \\
\hline Comparison $^{3)}$ & 3 & 0.88 & 2.37 & 2.74 & 2.97 & 1.32 & 1.13 \\
\hline Residual $^{4)}$ & $180-198$ & 2.30 & 2.28 & 2.28 & 2.11 & 2.32 & 2.16 \\
\hline Grand residual & $248-271$ & 2.93 & 2.87 & 2.87 & 2.72 & 2.85 & 2.69 \\
\hline
\end{tabular}

1) df varied according to the number of RILs with available marker data.

2) Mean squares listed are actual values $\times 10^{-2}$.

3) Variance source explained as an orthogonal comparison between marker genotypes, Asominori-type and IR24-type, within the variance source for RIL or for Interaction.

4) Variance source not explained as the comparison within the source for RIL or for Interaction.

**: indicates that the mean square is significant at the 0.01 probability level.

of the QTL distribution for different traits. Four genome regions on three chromosomes showed significant main effects for the total number of spikelets per plant. For one of the two component traits, number of spikelets per panicle, there were six significant regions, while for the other component, number of panicles per plant, only one. Region 28 on chromosome 6 showed a QTL for the number of spikelets per plant and its component, number of spikelets per panicle.

The distribution of both components of spikelets per panicle, namely number of spikelets on PBs and number of spikelets on SBs, suggested the existence of many significant genome regions. Among the six regions characterized by the number of spikelets per panicle, three $(1,21$ and 31) also showed significant effects for the number of spikelets on SBs, while only one (18) did so for the number of spikelets on PBs.

Ten genome regions exhibited significant main effects for the number of spikelets on PBs. Eight of these regions also showed a QTL for the number of PBs per panicle, one of the components of the number of spikelets on PBs, while the other component, number of spikelets per PB, did not show any significant effects in the above ten regions. Similarly, five significant regions out of six for the number of spikelets on SBs showed a significance for the number of SBs per panicle, while only two of them did so for the number of spikelets per SB. The numbers of SBs per panicle and of spikelets per SB shared one significant region (3).

\section{Genetic correlation}

The results of the analysis of genetic correlation (Table 4) were mostly consistent with the coincidence profiles of QTL main effects (Fig. 1 and Table 3). For example, the number of spikelets per panicle showed a higher genetic correlation with the number of spikelets on SBs $\left(r_{g}=0.93\right)$ than with the number of spikelets on PBs (0.33); the number of spikelets on PBs was highly correlated with the number of PBs per panicle (0.93) but not with the number of spikelets per PB (0.31); and the number of spikelets on SBs showed an extremely high correlation with the number of SBs per panicle (0.99) and a high correlation with the number of 

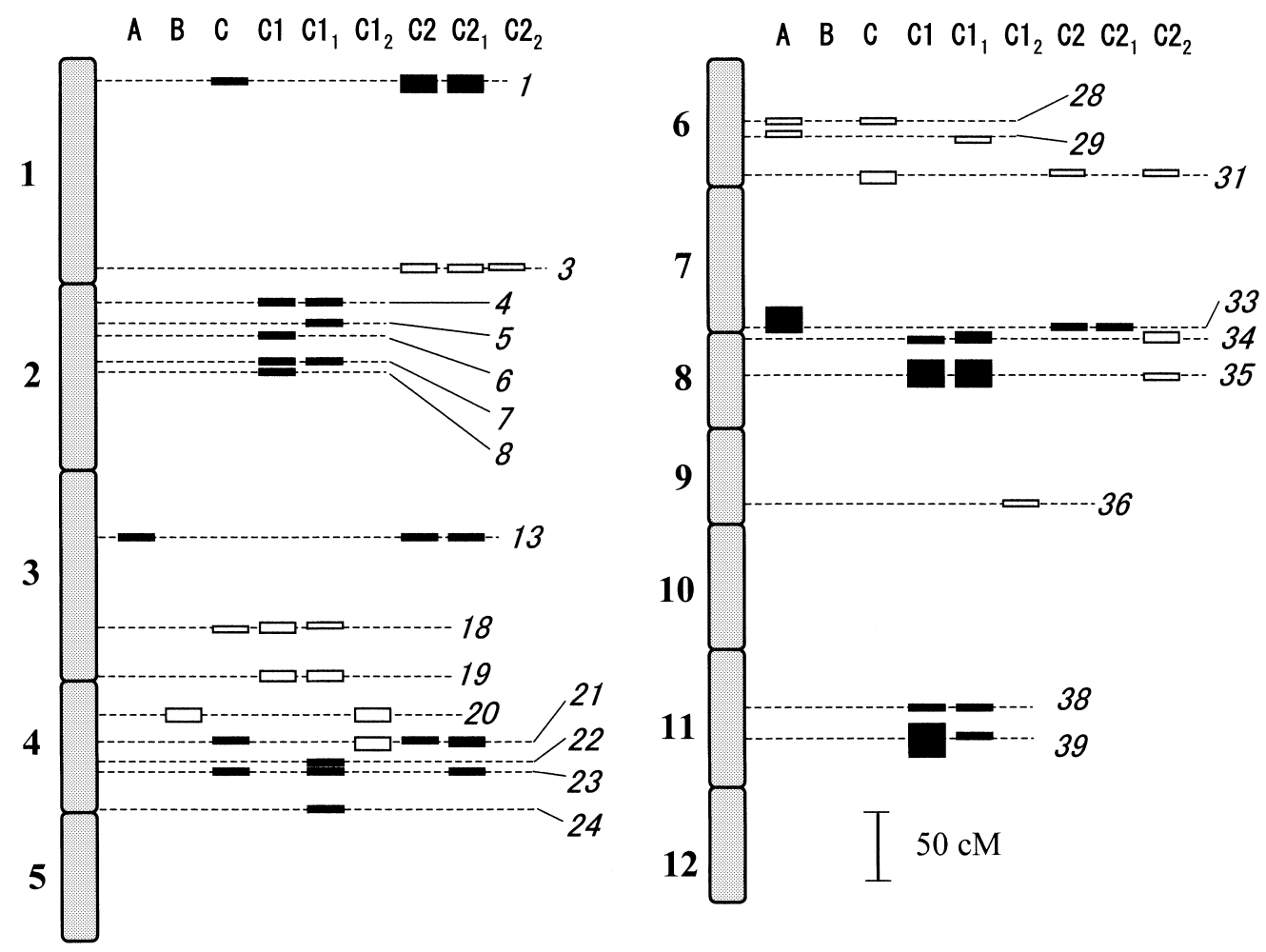

Fig. 1. Rice genome regions showing significant F-values $(P=0.01)$ in the ANOVA for the main effects for the number of spikelets per plant and its components, using the RILs of Asominori/IR24 grown over a period of four years.

A, number of spikelets per plant; $\mathrm{B}$, number of panicles per plant; $\mathrm{C}$, number of spikelets per panicle; $\mathrm{C} 1$, number of spikelets on $\mathrm{PBs} ; \mathrm{Cl}_{1}$, number of $\mathrm{PBs}$ per panicle; $\mathrm{Cl}_{2}$, number of spikelets per $\mathrm{PB}$; 2 , number of spikelets on $\mathrm{SBs}$; $\mathrm{C}_{1}$, number of $\mathrm{SBs}$ per panicle; $\mathrm{C}_{2}$, number of spikelets per SB. Halftone bars and attached bold numbers indicate rice linkage groups and their numbers, respectively. Rectangles indicate the marker positions or marker intervals showing significant F-values for individual traits. Italic numbers assigned at single or multiple rectangles indicate the region numbers cited in Table 3. Closed and open rectangles indicate that RILs with the marker genotype Asominori (closed) and IR24 (open), respectively, showed a significantly higher value than the counterpart for this trait in these markers.

spikelets per SB (0.73). Based on Figure 1 and Table 3, more regions tended to be shared by two traits showing higher genetic correlations, compared with the other traits showing lower genetic correlations, as discussed above. All of the correlation coefficients with absolute values higher than 0.4 were positive. This was consistent with the fact that the directions of QTL main effects of the correlated traits were the same, which could account for the positive coefficient of correlation.

\section{QY interaction}

Figure 2 and Table 5 show the detected QY interactions examined at every marker position. They suggested that many genome regions displayed significant QY interactions for all of the traits, in contrast to the results from the overall ANOVA (Table 1), in which significant interactions between most of the traits had not been revealed. This discrepancy could be attributed to the canceling out of the QY interactions showing opposite directions for the same trait. Four regions showed a significant $\mathrm{QY}$ interaction for the number of spikelets per plant. One of the four regions also showed a significance for one of the components, number of panicles per plant, while two of the four regions did so for the other component, number of spikelets per panicle. Moreover, for the number of spikelets per panicle there were seven significant regions only for this trait. Six out of the nine regions with a significant QY interaction for the number of spikelets per panicle were shared with one of the components of this trait (number of spikelets on SBs), whereas no coincident region was detected for the other one (number of spikelets on PBs).

For the number of spikelets on PBs, two regions with a significant QY interaction out of seven regions corresponded to the regions for the number of PBs per panicle, while no such regions were detected for the number of spikelets per PB. Five out of eight significant regions for the number of spikelets on SBs were shared with one of its components (number of SBs per panicle), and two with the other components (number of spikelets per SB). In no cases, did both components simultaneously share common significant 
Table 3. Significant F-values $(P=0.01)$ detected in the ANOVA for the main effects for the number of spikelets per plant and its components, using the RILs of Asominori/IR24 grown over a period of four years

\begin{tabular}{|c|c|c|c|c|c|c|c|c|c|c|c|}
\hline $\begin{array}{l}\text { Chromo- } \\
\text { some }\end{array}$ & Region & Marker ${ }^{1)}$ & $\mathrm{A}^{2)}$ & $\mathrm{B}$ & $\mathrm{C}$ & $\mathrm{C} 1$ & $\mathrm{C} 1_{1}$ & $\mathrm{Cl}_{2}$ & $\mathrm{C} 2$ & $\mathrm{C} 2_{1}$ & $\mathrm{C} 2_{2}$ \\
\hline \multirow[t]{2}{*}{1} & 1 & R3203-C86 & & & 10.8 & & & & 12.0 & 11.7 & \\
\hline & 3 & R3192-C970 & & & & & & & -11.1 & -10.3 & -9.6 \\
\hline \multirow[t]{5}{*}{2} & 4 & Кy6-C560 & & & & 10.7 & 9.1 & & & & \\
\hline & 5 & C601 & & & & & 7.2 & & & & \\
\hline & 6 & R3393 & & & & 8.1 & & & & & \\
\hline & 7 & C920-XNpb67 & & & & 15.8 & 11.9 & & & & \\
\hline & 8 & $\mathrm{XNpb45}$ & & & & 9.4 & & & & & \\
\hline \multirow[t]{3}{*}{3} & 13 & C1351 & 13.6 & & & & & & 7.2 & 8.2 & \\
\hline & 18 & XNpb392-C563 & & & -8.4 & -12.4 & -12.1 & & & & \\
\hline & 19 & $\mathrm{XNpb} 279-\mathrm{C} 515$ & & & & -10.4 & -10.0 & & & & \\
\hline \multirow[t]{5}{*}{4} & 20 & R2737-R2783 & & -7.5 & & & & -19.9 & & & \\
\hline & 21 & C335-XNpb49 & & & 9.0 & & & -25.5 & 9.7 & 14.8 & \\
\hline & 22 & $\mathrm{XNpb271-C140}$ & & & & & 8.8 & & & & \\
\hline & 23 & $\mathrm{C} 975$ & & & 8.7 & & 8.9 & & & 7.9 & \\
\hline & 24 & $\mathrm{R} 2373$ & & & & & 7.2 & & & & \\
\hline \multirow[t]{3}{*}{6} & 28 & R1985B-C282B & -9.7 & & -7.3 & & & & & & \\
\hline & 29 & XNpb172-C1677B & -9.5 & & & & -7.2 & & & & \\
\hline & 31 & C259C-XNpb170 & & -9.0 & & & & & -8.2 & & -8.5 \\
\hline 7 & 33 & R1789-C728 & 17.0 & & & & & & 8.2 & 7.2 & \\
\hline \multirow[t]{2}{*}{8} & 34 & C83-XNpb56 & & & & 11.8 & 16.7 & & & & -11.4 \\
\hline & 35 & R2382-C483 & & & & 12.5 & 16.3 & & & & -7.2 \\
\hline 9 & 36 & C609 & & & & & & -7.2 & & & \\
\hline \multirow[t]{2}{*}{11} & 38 & C1172 & & & & 7.9 & 7.1 & & & & \\
\hline & 39 & $\mathrm{R} 728-\mathrm{C} 1350$ & & & & 10.9 & 8.5 & & & & \\
\hline
\end{tabular}

1) The longest marker intervals are cited when the relevant regions involved multiple markers.

2) A, number of spikelets per plant; $\mathrm{B}$, number of panicles per plant; $\mathrm{C}$, number of spikelets per panicle; $\mathrm{C} 1$, number of spikelets on PBs; $\mathrm{Cl}_{1}$, number of PBs per panicle; $\mathrm{Cl}_{2}$, number of spikelets per $\mathrm{PB} ; \mathrm{C} 2$, number of spikelets on $\mathrm{SBs} ; \mathrm{C}_{1}$, number of $\mathrm{SBs}$ per panicle; $\mathrm{C}_{2}$, number of spikelets per $\mathrm{SB}$.

The largest significant F-values are cited when a region involved multiple markers. + and - on an F-value indicate that RILs with the marker genotype Asominori(+) and IR24(-), respectively, showed a significantly higher value than the counterpart for this trait in this region. The critical F-value for significance at the 0.01 probability level is about 7.03 .

Table 4. Genetic correlation coefficients between traits related to rice spikelet number per plant, using RILs of Asominori/IR24 grown over a period of four years

\begin{tabular}{|c|c|c|c|c|c|c|c|c|}
\hline Trait & A & B & $\mathrm{C}$ & $\mathrm{C} 1$ & $\mathrm{Cl}_{1}$ & $\mathrm{Cl}_{2}$ & $\mathrm{C} 2$ & $\mathrm{C} 2_{1}$ \\
\hline Number of spikelet plant ${ }^{-1}$ (A) & & & & & & & & \\
\hline Number of panicles plant ${ }^{-1}(\mathrm{~B})$ & 0.50 & & & & & & & \\
\hline Number of spikelets panicle ${ }^{-1}(\mathrm{C})$ & 0.60 & -0.38 & & & & & & \\
\hline Number of spikelets on PBs (C1) & -0.01 & -0.36 & 0.33 & & & & & \\
\hline Number of PBs panicle ${ }^{-1}\left(\mathrm{Cl}_{1}\right)$ & 0.06 & -0.36 & 0.41 & 0.93 & & & & \\
\hline Number of spikelets $\mathrm{PB}^{-1}\left(\mathrm{Cl}_{2}\right)$ & -0.19 & -0.05 & -0.18 & 0.31 & -0.05 & & & \\
\hline Number of spikelets on SBs (C2) & 0.63 & -0.26 & 0.93 & -0.03 & 0.09 & -0.31 & & \\
\hline Number of SBs panicle ${ }^{-1}\left(\mathrm{C} 2_{1}\right)$ & 0.63 & -0.27 & 0.95 & 0.03 & 0.16 & -0.33 & 0.99 & \\
\hline Number of spikelets $\mathrm{SB}^{-1}\left(\mathrm{C}_{2}\right)$ & 0.42 & -0.15 & 0.56 & -0.36 & -0.36 & -0.03 & 0.73 & 0.63 \\
\hline
\end{tabular}

regions with their compound traits in terms of the QY interaction (Fig. 2 and Table 5), in contrast to the results from the main effect analysis (Fig. 1 and Table 3 ).

\section{Discussion}

\section{QTLs for spikelet number}

In this study, the QTL main effects and QY interactions in the rice genome for traits related to the number of rice spikelets per plant were estimated. An ANOVA was employed in this study instead of interval mapping to identify the genome regions of interest. On the other hand, many of the main effects detected in this study were consistent with the findings obtained in relevant studies using the interval mapping technique. Ando et al. (2001) detected a QTL, $q S N-1$, on the short arm of chromosome 1 in a mapping population that differed from the present one. The allele from an indica cultivar, Habataki, at $q S N-1$ appeared to increase the 

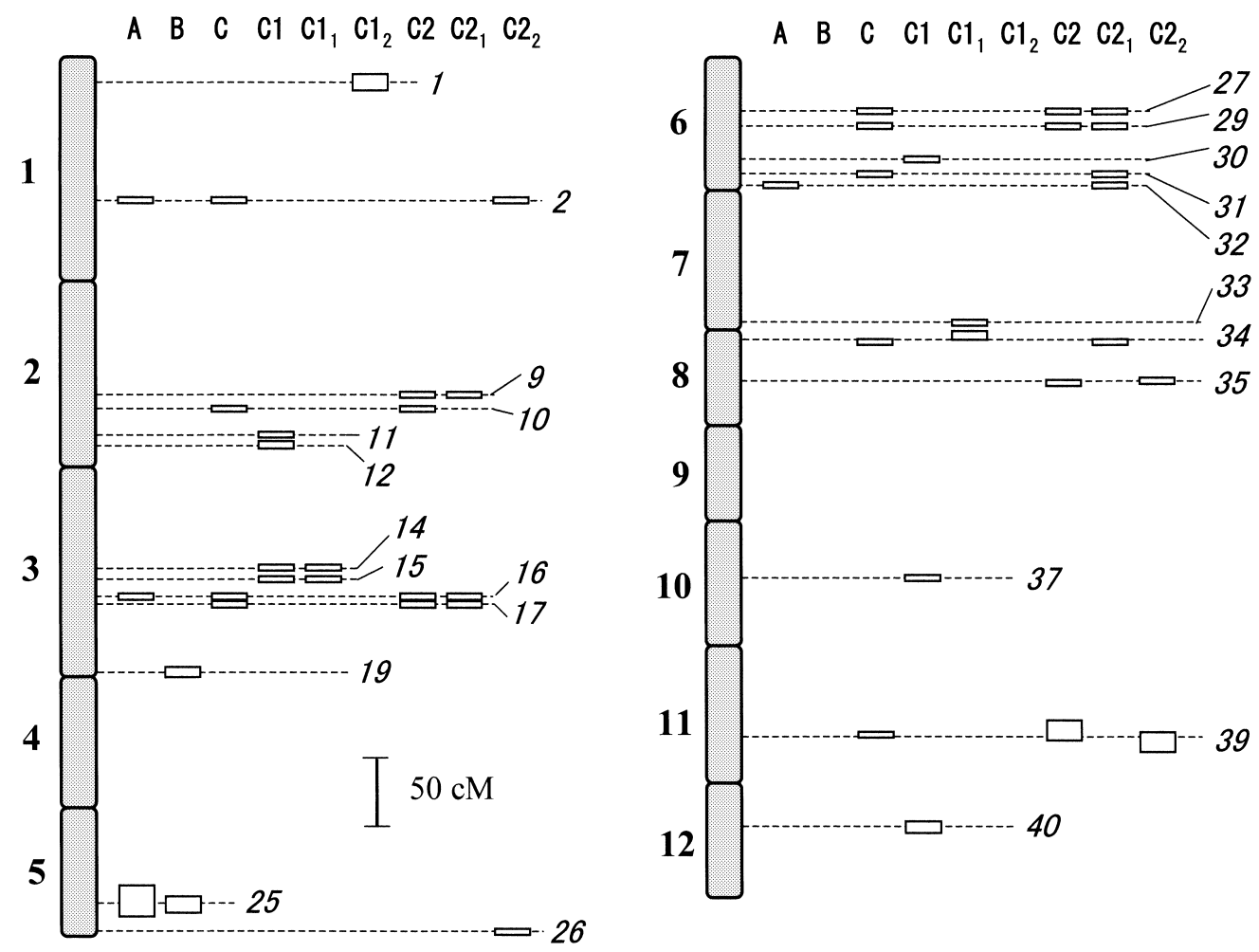

Fig. 2. Rice genome regions showing significant F-values $(P=0.01)$ in the ANOVA for the QY interactions of the number of spikelets per plant and its components, using the RILs of Asominori/IR24 grown over a period of four years.

$\mathrm{A}$, number of spikelets per plant; $\mathrm{B}$, number of panicles per plant; $\mathrm{C}$, number of spikelets per panicle; $\mathrm{C} 1$, number of spikelets on $\mathrm{PBs} ; \mathrm{Cl}_{1}$, number of $\mathrm{PBs}$ per panicle; $\mathrm{Cl}_{2}$, number of spikelets per $\mathrm{PB} ; \mathrm{C} 2$, number of spikelets on $\mathrm{SBs} ; \mathrm{C}_{1}$, number of SBs per panicle; $\mathrm{C}_{2}$, number of spikelets per SB. Halftone bars and attached bold numbers indicate rice linkage groups and their numbers, respectively. Rectangles indicate the marker positions or marker intervals showing significant F-values for individual traits. Italic numbers assigned at single or multiple rectangles indicate the region numbers cited in Table 5 .

number of spikelets per panicle by increasing the numbers of SBs and spikelets on each SB. Markers linked to the $q S N-1$ were located within the region 3 identified in this study. Although region 3 did not show a significant increase in the spikelet number per panicle, it significantly increased the numbers of spikelets on SBs, SBs per panicle and spikelets per SB. Also, the chromosome segment in this region was derived from an indica cultivar, IR24. Therefore, $q S N-1$ seems to correspond to the QTL assumed to exist in region 3. Yagi et al. (2001) and Nagata et al. (2002) also detected QTLs with strong effects (LOD scores of more than 8.0) on the number of spikelets per panicle and the number of SBs at almost the same position as region 3 in another mapping population derived from the cross of a japonica-cultivar Akihikari and an indica-cultivar Milyang 23.

Sasahara et al. (1999) mapped several QTLs controlling the number of PBs in the same mapping population as that used in the present study by interval mapping. Six of their QTLs (qNRB-2-1, qNRB-4-2, qNRB-8-1, qNRB-8-2, $q N R B-8-3$ and $q N R B-8-4$; Sasahara et al. 1999) out of eight appeared to be equivalent to the genome regions identified in the present study, in terms of their positions and additive effects. $q N R B-2-1, q N R B-4-2$ and $q N R B-8-1$ were present in the regions 7,23 and 34 , respectively. The other three $(q N R B-8-2, q N R B-8-3$ and $q N R B-8-4)$ were located in region 35 . It can be concluded that the present ANOVA procedure was suitable for the detection of genome regions probably involving QTLs, at least in the present case, and also for the detection of QY interaction simultaneously.

\section{Genetic association of main effects}

In region 3, the main effect on the number of spikelets per panicle appeared to be due to one of its components, number of spikelets on SBs, and also to its sub-component, number of SBs per panicle. At least one QTL in this region, probably $q S N-1$, must be responsible for the genetic association of those traits, resulting in extremely high genetic correlations $\left(r_{g}=0.93\right.$ between the numbers of spikelets per panicle and spikelets on SBs, 0.99 between the numbers of spikelets on SBs and SBs per panicle, and 0.95 between the numbers of spikelets per panicle and SBs per panicle).

As mentioned above, Kato and Takeda (1996) pro- 
Table 5. Significant F-values ( $P=0.01$ ) detected in the ANOVA for the QY interactions of the number of spikelets per plant and its components, using the RILs of Asominori/IR24 grown over a period of four years

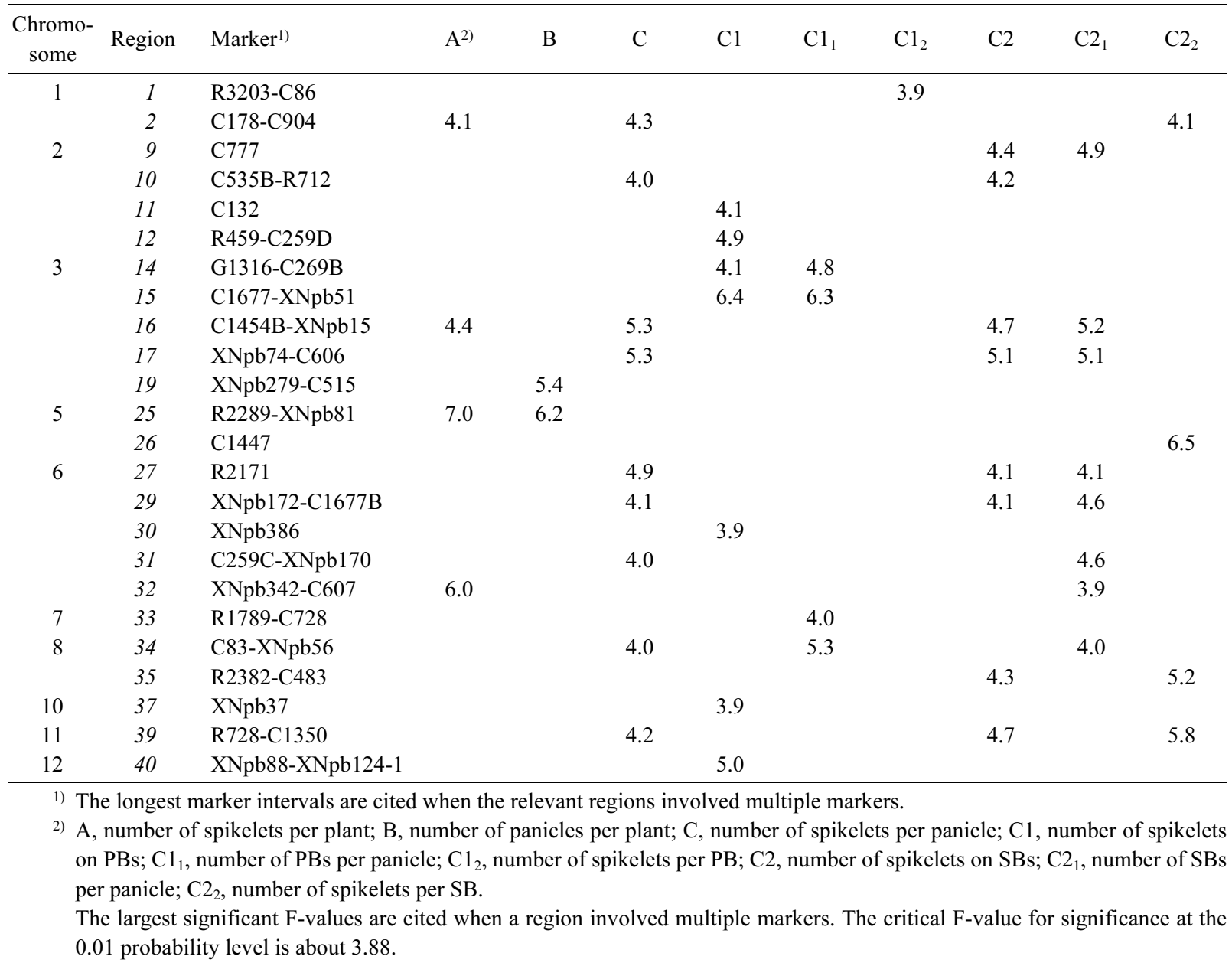

posed an ideal extra-heavy panicle rice whose spikelet number should be raised primarily by increasing the number of spikelets on PBs. In the present study, one genome region (18) on chromosome 3 was identified as a candidate gene source relating to this ideal panicle type. Region 18 was associated with the increase in the number of PBs per panicle, resulting in the increase in the numbers of spikelets on PBs and spikelets per panicle. It should be noted that the analysis did not provide any indication that this region might also be associated with the increase of production of spikelets on SBs, which usually show a low degree of grain filling. Although the additive effect of this region has not been evaluated precisely, it would be worthwhile to isolate and utilize this region, or the QTL(s) probably involved in this region.

\section{Interactions of QTL and year of cultivation for different traits}

Using the approach presented in this study, interactions of QTL and year of cultivation detected in a compound trait could be assigned to one of the trait components. Such an assignment suggested that the QY interaction regarding the number of spikelets per panicle was due to its component, number of spikelets on SBs, and also to the sub-component of the latter, number of SBs per panicle. Only three traitregion combinations out of 47 in which significant interactions were detected (Table 5), showed also significant main effects. This suggests that a QY interaction of a crossover type was prevailing in the traits examined. Regarding the region 18 discussed above, no interaction was observed, suggesting that the QTL(s) within this region should remain stable over years.

Frequently, multi-trial experiments using QTL analysis have been conducted to characterize quantitative traits. In most of these studies, the QTL-by-environment interactions detected were evaluated by comparing the LOD-scores of the regions of interest between all the environmental conditions examined (Zhuang et al. 1997, Austin and Lee 1998, Nagata et al. 2002). On the other hand, several models and procedures have been developed to detect and map the genotype-by-environment interaction onto the genome (Hayes et al. 1993, Jansen et al. 1995, Romagosa et al. 1996, Wang et al. 1999). In the present study, ANOVA was used to detect QY interactions at each marker position, and the coincidence profile of the genome regions responsible for 
significant QY interactions concerning different traits was examined. This approach might provide another useful tool to analyze the genotype-by-environment interaction for a compound trait such as grain yield.

\section{Acknowledgements}

The author greatly appreciates the support extended for this study by K. Keito, J. Sako, M. Nakata, Y. Ogushi, Y. Sato, A. Fujii, M. Sugiura and Dr. T. Itani, Hiroshima Prefectural University, Japan. The author also wishes to thank Drs. K. Takeda and K. Sato, Okayama University, Japan, and Dr. P.K. Mohapatra, Sambalpur University, India, for their critical reading of the manuscript.

\section{Literature Cited}

Ando,T., S.Y.Lin, A.Shomura, T.Shimizu and M.Yano (2001) RFLP mapping of gene controlling seed number per panicle on chromosome 1 in rice. Breed. Res. 3 (Suppl. 2): 44 (in Japanese).

Austin,D.F. and M.Lee (1998) Detection of quantitative trait loci for grain yield and yield components in maize across generations in stress and nonstress environments. Crop Sci. 38: 1296-1308.

Feil,B. (1992) Breeding progress in small grain cereals-A comparison of old and modern cultivars. Plant Breed. 108: 1-11.

Hayes, P.M., B.H.Liu, S.J.Knapp, F.Chen, B.Jones, T. Blake, J. Franckowiak，D. Rasmusson，M. Sorrells， S.E. Ullrich, D.Wesenberg and A.Kleinhofs (1993) Quantitative trait locus effects and environmental interaction in a sample of North American barley germplasm. Theor. Appl. Genet. 87: 392-401.

Jansen,R.C., J.W.Van Ooijen, P.Stam, C.Lister and C.Dean (1995) Genotype-by-environment interaction in genetic mapping multiple quantitative trait loci. Theor. Appl. Genet. 91: 33-37.

Kato,T. (1996) Diallel analyses of the number of spikelets per plant and its components in rice (Oryza sativa L.). SABRAO Journal 28: $1-6$.

Kato,T. (1997) Selection responses for the characters related to yield sink capacity of rice. Crop Sci. 37: 1472-1475.

Kato,T. and K.Takeda (1996) Associations among characters related to yield sink capacity in space-planted rice. Crop Sci. 36: 11351139.
Khush,G.S. (1996) Prospects and approaches to increasing the genetic yield potential of rice. In "Rice Research in Asia. Progress and Priorities" Everson, R.E. et al. (eds.), CAB International, Wallingford, p. 59-71.

Nagata,K., Y.Fukuta, H.Shimizu, T.Yagi and T.Terao (2002) Quantitative trait loci for sink size and ripening traits in rice (Oryza sativa L.). Breed. Sci. 52: 259-273.

Peng,S., K.G.Cassman, S.S.Virmani, J.Sheehy and G.S.Khush (1999) Yield potential trends of tropical rice since the release of IR8 and the challenge of increasing rice yield potential. Crop Sci. 39: 1552-1559.

Romagosa,I., S.E.Ullrich, F.Han and P.M.Hayes (1996) Use of the additive main effects and multiplicative interaction model in QTL mapping for adaptation in barley. Theor. Appl. Genet. 93: 3037.

Sasahara,H., Y.Fukuta and T.Fukuyama (1999) Mapping of QTLs for vascular bundle system and spikelet morphology in rice. Breed. Sci. 49: 75-81.

Snedecor,G.W. and W.G.Cochran (1967) Statistical Methods (6 $6^{\text {th }}$ ed.). Iowa Univ. Press, Ames, Iowa, p. 339-380.

Tsunematsu,H., A.Yoshimura, Y.Harushima, Y.Nagamura, N.Kurata, M.Yano, T.Sasaki and N.Iwata (1996) RFLP framework map using recombinant inbred lines in rice. Breed. Sci. 46: 279-284.

Wang,D.L., J.Zhu, Z.K.Li and A.H.Paterson (1999) Mapping QTLs with epistatic effects and QTL $\times$ environment interactions by mixed linear model approaches. Theor. Appl. Genet. 99: 12551264.

Yagi,T., K.Nagata, Y.Fukuta, K.Tamura, I. Ashikawa and T.Terao (2001) QTL mapping of spikelet number in rice (Oryza sativa L.). Breed. Sci. 51: 53-56.

Yamamoto, Y., T.Yoshida, T.Enomoto and G.Yoshikawa (1991) Characteristics for the efficiency of spikelet production and the ripening in high-yielding japonica-indica hybrid and semidwarf indica rice varieties. Jpn. J. Crop Sci. 60: 365-372 (in Japanese with English summary).

Yang,J., S.Peng, Z.Zhang, Z.Wang, R.M.Visperas and Q.Zhu (2002) Grain and dry matter yields and partitioning of assimilates in japonica/indica hybrid rice. Crop Sci. 42: 766-772.

Zhuang,J.Y., H.X.Lin, J.Lu, H.R.Qian, S.Hittalmani, N.Huang and K.L.Zheng (1997) Analysis of QTL $\times$ environment interaction for yield components and plant height in rice. Theor. Appl. Genet. 95: 799-808. 